\title{
Modeling and Evaluation of Intelligent Monitoring Systems at Urban Intersections to Improve Real-Time Monitoring of Congestion and Traffic Safety
}

\author{
Negin Fatholahzadeh \\ Department of Computer \\ Engineering \\ Islamic Azad University \\ E-Campus \\ Tehran, Iran
}

\author{
Gholamreza Akbarizadeh \\ Department of Electrical \\ Engineering, Faculty of \\ Engineering \\ Shahid Chamran University of \\ Ahvaz \\ Ahvaz, Iran
}

\author{
Morteza Romoozi \\ Department of Computer \\ Engineering \\ Kashan Branch, Islamic Azad \\ University \\ Kashan, Iran
}

\begin{abstract}
In recent decades, with the advent of technology and information technology, transportation systems have also been moving in this direction, and one of the challenges of the transportation system is due to the multiplicity and increase of vehicle management and control. Many techniques and methods have been proposed in this field that in today's world, the use of intelligent systems has a higher efficiency. In fact, safety has the ability to reduce the number of vehicles with minimal delay and create discomfort for the users depends directly in order and arrangement to facilitate the immediate and direct involvement of the vehicle in traffic flow. One of the ways to demonstrate the efficiency of intelligent monitoring systems is create model before the implementation phase.

This article aims at modeling and evaluating an intelligent surveillance system at urban intersections, which is carried out using colored petri nets. In the proposed method, first, the urban intersection architecture is described with the benefit of traffic rules then, the inconsistency and coincidence states are determined and finally, reliability and response time are measured by the CpnTools with the help of Petri Nets. The results show that the intelligent monitoring equipment related to the intelligent surveillance system increases the safety of the road network and, finally, has secured and fluent traffic.
\end{abstract}

Keywords: Sydney Coordinated Adaptive Traffic System; Urban Intersections; Colored Petri Nets; Reliability; Response time.

\section{INTRODUCTION}

In the last few decades significant progress there have been in the field of intelligent technologies. While the introduction of the new system to provide accurate and qualitative readings of the up-to-date system, the use of these systems is always worth Investigate in the efficiency of traffic and safety. Many of these studies have examined the total and non-total effects of traffic flow-rate parameters on the collision event. Therefore, based on the current findings, there is no experimental study on the effect of traffic congestion on the safety of the vehicle. In addition, the congestion as one of the ordinary traffic phenomena in the crossover city itself only affects its own effect on the safety of the road also it acts as a direct criterion. As a result, the traffic safety and efficiency of it are interconnected. There is a need for testing each one and examining the potential for improvement both at the same time, especially with the availability of traffic discovery data.

Nowadays, the use of modeling methods in industrial works, has found wide application especially with the development of advanced sciences and increasing the speed of processors. One of the modeling uses the petri nets. The goal of the modeling is to study and evaluate the reference system. The basis of the modeling is the selection of a suitable model. Choosing the appropriate model is a determinant parameter, so firstly, the model should be well understood. Any kind of presentation or expression of a system is called a model. The model describes the behavior of the system and allows for the implementation, simplification, and the creation of uniformity and uniqueness. One of the methods of modeling is to use a petri nets.

Petri nets modeling have an important advantage that can show coherence in a comprehensive and graphical way. In fact, the study system of this paper, which is an urban intersection, is in line with this requirement, and we can evaluate intelligent monitoring systems. And correctly understand their function and provide accurate information and reports to decision makers with the required conditions in order to be more precise in making important decisions. In fact, two things are done with modeling, one is that the function of the intelligent monitoring systems embedded at the intersections is measured and evaluated. And from another perspective, it can be used to equip new intersections whether the target system could manage the intersection in critical conditions to control congestion and traffic? This activity is also carried out before the implementation phase and is a significant contribution to cost savings.

The structure of the article is as follows: In Section 2, fundamental concepts are explained such as Sydney Coordinated Adaptive Traffic System, urban intersections, colored petri nets, reliability and response time. In Section 3, a useful selection of previous studies is described in brief similar to the subject matter. And fourth section, is described the proposed method. Finally, to demonstrate the validity of the proposed method in Section 5, a case study is presented to evaluate and modeling the proposed method.

\section{FUNDAMENTAL CONCEPTS}

In each research, researchers work according to a particular viewpoint on topics. In this paper, the definitions are used due to different definitions of concepts, the type of application and their efficiency, and in the following describes these concepts.

\subsection{Sydney Coordinated Adaptive Traffic} System

The most important feature of this system, adapted for use in different parts of the world and with different and relatively contradictory traffic cultures, is the momentary and 
completely adaptive response to traffic changes at each intersection, taking into account the traffic of the associated arteries. Unlike other traffic control systems, the SCATS instantly generates LED traffic parameters and adjusts the timing of lights based on traffic flow and saturation in each cycle. While other systems use predefined traffic models, they are sometimes not adapted to local traffic drivers' behavior [12].

The system is capable of responding to the momentary changes in demand and capacity and automatically adjusts the timing of the lights so that the traffic flow is optimized at the network level. Traffic volume and the efficiency of each intersection are measured by vehicle sensors such as visual, radar, and inductive loops and the best timing is selected and applied for optimizing intersection schedules. Unlike other control methods, this system does not use mathematical and theoretical models to allocate optimal scheduling because the traffic behavior of drivers in different parts of the world is different and the use of traffic models creates limitations. SCATS are a modular system and therefore its development is possible. Its structure is hierarchical, and if the controller's connection to the center is disconnected, in addition to reporting the error to the center, the controller will enter a separate operation mode and act individually and in accordance with its predetermined schedule. Therefore, system operation continues in any situation.

With the use of the SCATS central control system, it is possible to monitor the performance of all invertebrate controllers from a centralized location, and in the event of any failure, such as vehicle sensors, bulbs, or communication line failure with the controller an intersection, a message sent about the central computer, and it is possible to quickly dispatch the maintenance personnel to repair the related equipment.

\subsection{Urban Intersections}

The intersection or the crossroads of the crossing point are two ways. Breakouts are an inevitable part of the urban road network. As many urban streets share at least one intersection. According to studies, a large proportion of traffic accidents occur at intersections. The main cause of these crashes at intersections is the convergence of different traffic flows at a point. Accident at intersections can be due to different factors, each of which requires a suitable approach [10].

The important thing is that the capacity of each link and, in general, the capacity of the city's traffic network depends on the capacity and the ability to pull the intersections of that link or network. The capacity of the intersections determines the capacity of urban roads. In analyzing intersections, we are facing with three intersections of the same level in urban roads;

Intersections that used the timed traffic lights in them. At these intersections, the intersection is controlled by flashing traffic lights or traffic signs and cannot display any signs. These intersections are referred to subway intersection. Intersections controlled by a signalized traffic light. Square, intersection of a circular, one-way, and without traffic lights equipment, in which the traffic flow moves around a circular island to separate vehicles that travel in different directions and to reduce the number of collisions and also better traffic guidance is trying to separate the intersections of the level [8].

\subsection{Colored Petri-Nets}

Provides a graphical representation of the system with a mathematical approach, and they can illustrate communication patterns, control patterns, and information flows. These networks provide a framework for analysis, validation and performance evaluation. The basis of the Petribased nets is on the graph, and informally it can be said that a two-part directed graph that are formed two elements of location and transition. These networks are based on a situation, not an event, which makes the model explicit the status of each item. Petri Nets offer models of structural and behavioral aspects of a discrete event. It also provides a framework for analyzing, validating and evaluating performance and reliability [7].

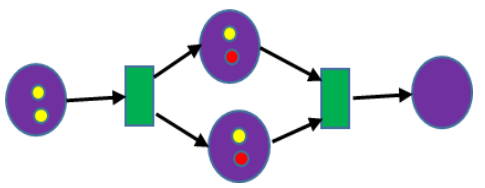

Figure 1. A model of a colored petri nets

Petri nets (see Figure 1) provide more precise models of nonsynchronous processing systems. In these networks, unlike petri nets, the beads are distinct from each other because each of the beads has attributes called colors. These types of networks make it possible to make a more detailed and detailed modeling of the asynchronous processes. The arcs can contain mathematical expressions composed of a combination of color sets and their related variables. Guard is a Boolean expression that is attributed to a transition and creates conditions for activating the input arc. In the petricolored net of each location, arrows and transfers can have their own guard depending on the color of them [6]. Title and Authors

\subsection{Reliability}

This feature in the networks refers to the existence of backup servers on the network, this means that you can back up from different sources of information and systems and provide secondary versions and backup, and in the absence of access to one of the resources on the network or to shut down a system used the backup copies. The system works correctly at the time interval $[\mathrm{t} 0, \mathrm{t}]$, provided that the system is correct at the beginning of the interval $(\mathrm{t} 0)$ and is expected to provide the service without interruption, such as spatial applications but in time system access (t) (whenever needed) is working properly and available and can perform its function. The system of banks can be expressed as an example [5].

\subsection{Response Time}

when we press the last key on the keyboard to see the result on the screen, or from the moment it runs a requested process or process until the moment when the processor requests the operating system, in other words, the amount of time it takes is to process a notice to the network, and the result will be delivered in response to the requester. (see Figure 2) shows that when a request is given to the system, the response time is up to the last time the request is received, it is the response time, and the user will be satisfied with the less time. In fact, the rapid operation of the system accelerates the traffic and congestion management at critical points with regard to the performance of drivers. And in this paper, this parameter is displayed on the Intelligent Traffic Monitoring Network using colored Petri Nets [4]. 


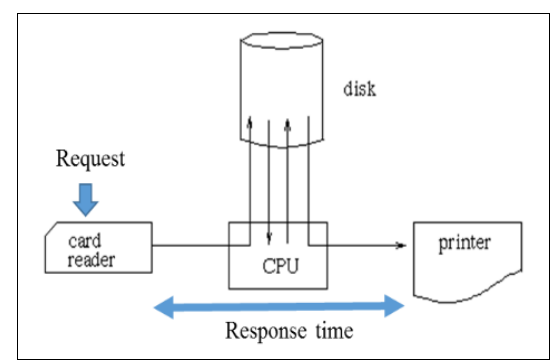

Figure 2. Request Response Time

\section{RELATED WORK}

In recent years, various methods have been developed to model and evaluate intelligent surveillance systems at intercity intersections in order to reduce congestion and traffic in real time. Different research methods have been used in these methods. Here are few items to consider in this article. In the modeling method, it has made it possible to have a seasonal variety, variations in the level of the unit of Collision, and the incidental effects of an intersectional surface have Support feature on the collision event. The design of this method is based on Bayesian Conclusion Techniques is represented that more unmatched heterogeneity can be recorded and the model will be better able to be categorized [1].

A random forest analysis was used to identify the major traffic inputs affecting the collision event is executed using AVI data on a CFX system [63]. In concluding that AVI data is presented a risk-based collision measure at the actual time, however, method providers point out that when they do - That the AVI parts are average of $1.5 \mathrm{~m}$ [11].

In another way, the related research is based on a research paper based on the findings of researchers that there is an overload in all cities of the world. They have a new algorithm for diagnosis. The project was designed in two aspects. One is the offline processing data and the other one online congestion management. Using the standard function method for calculating the parameters, were standardized the thresholds of the internal and external dimensions of the integrated circuit. The purpose of this action is to determine the quantity of each parameter, and the identification of the road congestion, and traffic congestion monitoring [14].

In another method, a genetic algorithm is proposed to solve the scheduling of related tasks, in which two important parameters of the quality of service are considered which are time and cost. In this algorithm, instead of producing the random initial population turbulent variable are used. The combine points of genetic algorithm ratings with turbulent variables have allowed the solutions generated by this algorithm to be distributed throughout the search space and prevent early convergence of the algorithm. Better designs and products are obtained in shorter time and to get it the algorithm converges to a faster rate [2].

In another method, related to the topic of research, based on the views of the researchers, the events of the metropolis are a metric key-performance-road, which is recognized as one of the key sources of non-recursive congestion. Traffic accidents are often terminated to reduce the travel time's promise. The proposed method is to determine the effect of traffic events on the reliability the travel time's in the freeway. Measurement of the reliability of these events indicates the amount of time it takes for passengers to travel and arrived their destination at a specified time, and more importantly, this is the travel time's should be recursive [3].

In another way, they were related to the subject of research, the researchers believed that the method of evaluating the effective traffic situation is very important, to get the behavior of the traffic system in the road. Based on this theory, they selected urban roads for studying and evaluated the actual situation in real time. In the first step, the situation was divided into six parts and the evaluation of the traffic was considered as an important problem in the classification. Then, this point was considered as a point of view of the traffic managers the speed velocity is selected as the evaluation index. On the basis of this, a new approach was developed for data rapid integration and the factors enterprises for evaluating the traffic situation. The effectiveness of the results is validated by Real-time data traffic [13].

In 2010, Dow and others provided the IGA algorithm to solve the problem of scheduling affiliated tasks, in which three parameters were considered simultaneously of quality of service. These three parameters are time, cost, and reliability. Because these parameters are in conflict with each other and cannot be simultaneously recovered, improving one will reduce other efficiency, is weighed each of the parameters. So that the weight is either made by the user so that each of the parameters that is more valuable to the user is more weight and the other is weighing less or that the weight is randomly done [9].

\section{SUGGESTED METHOD}

Urban intersections can be a big challenge considering the vital traffic hotspots in the city and the lack of accurate management in this area. And many solutions, including intelligent monitoring systems, are implemented in these sites, but the accuracy intelligent systems before the implementation phases to avoid spending a lot of costs and management in different circumstances required by the road In this study, we are using a four-way urban model with the aid of colored petri nets in order to have a thorough evaluation of reliability and response time, the proposed methodology is described below.

\subsection{Architecture Proposed Method}

Different sensors and cameras are embedded in four-way recorded the current data and sent them in the database. All the data archived due to their massive volume. (see Figure 3) shows an overview of the architecture of the proposed method. In the first case, a four-way was designed for evaluation with the consideration of the sensors location in different areas, the four-way is modeled using colored petri nets. But in the next step, we began to Collected the data in the model. As shown in (see Figure 3), these data are related to the freeway through the sensors recorded at the time and archived with all types of properties in the substrate. And then, for extraction of data, the characteristics of the object and their analysis are determined for traffic management by conducting and determining the path with time. In this case, the length of the paths over and above due to the traffic congestion so the traffic will be controlled fluently and the safety and non-crash will be managed in real time. 


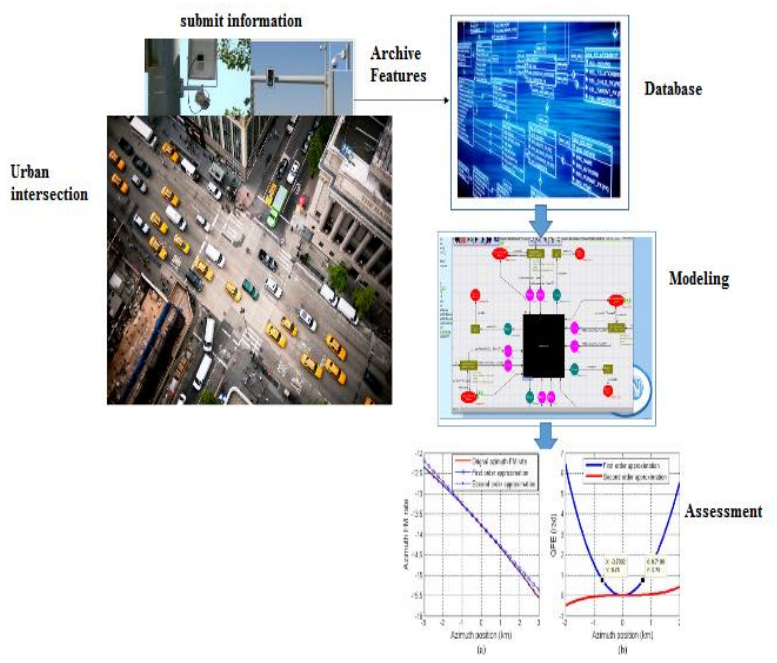

Figure 3. Architecture offered by the proposed method

\subsection{Urban Intersection Modeling}

Modeling and designing is now widely used in the industry. In fact, by modeling and designing, an industrial system is studied before its creation, and it's affordable economically feasible in terms of time. Petri nets make it possible the component and next to each other study of system Petri nets are appropriate method based on the mathematical logic graphically representation although it is in fact a graphical representation of the network, but the substrate Strong mathematical.

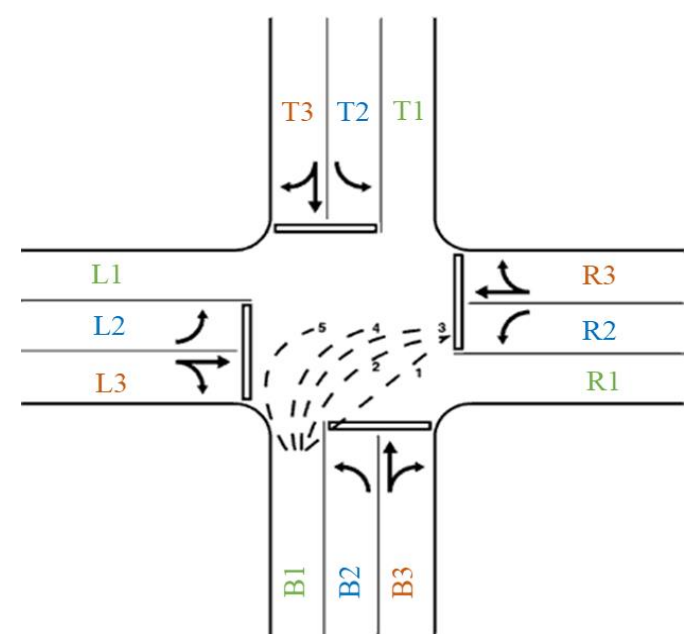

Figure 4 - Description of urban intersection rules

Each petri net using only four elements (location, transfer, arc, and bead), performs the modeling action. In order to reduce costs, we model it instead of using sensors and real-life cameras at the intersection of the city. Therefore, for modeling to be possible, we must describe the specified rules according to the real model. (see Figure 4). Table (1) shows the collision, concurrency, and Sustainable synchronization corresponding to (see figure 1)
Table 1. permitted and non- permissible behavior at the intersection

\begin{tabular}{|c|c|c|c|c|c|c|c|c|c|c|c|}
\hline \multicolumn{3}{|c|}{ Concurrency } & \multicolumn{3}{|c|}{ Concurrency Stable } & \multicolumn{6}{|c|}{ Incompatibility } \\
\hline $\mathrm{T} 3$ & $\approx$ & B3 & T3 & $\cong$ & $\mathrm{T} 2$ & T3 & $\infty$ & L3 & $\mathrm{T} 2$ & $\infty$ & R3 \\
\hline $\mathrm{T} 3$ & $\approx$ & $\mathrm{T} 2$ & $\mathrm{~L} 2$ & $\cong$ & L3 & T3 & $\infty$ & R3 & $\mathrm{T} 2$ & $\infty$ & L2 \\
\hline R3 & $\approx$ & L3 & B3 & $\cong$ & B2 & T3 & $\infty$ & L2 & $\mathrm{T} 2$ & $\infty$ & R2 \\
\hline R3 & $\approx$ & $\mathrm{R} 2$ & R3 & $\cong$ & R2 & T3 & $\infty$ & B2 & $\mathrm{T} 2$ & $\infty$ & B3 \\
\hline B3 & $\approx$ & $\mathrm{T} 3$ & & & & B3 & $\infty$ & $\mathrm{T} 3$ & L2 & $\infty$ & T3 \\
\hline B3 & $\approx$ & $\mathrm{B} 2$ & & & & B3 & $\infty$ & R3 & $\mathrm{L} 2$ & $\infty$ & R3 \\
\hline L3 & $\approx$ & R3 & & & & B3 & $\infty$ & L3 & L2 & $\infty$ & $\mathrm{T} 2$ \\
\hline L3 & $\approx$ & L2 & & & & B3 & $\infty$ & $\mathrm{R} 2$ & L2 & $\infty$ & B2 \\
\hline $\mathrm{T} 2$ & $\approx$ & $\mathrm{T} 3$ & & & & B3 & $\infty$ & $\mathrm{T} 2$ & $\mathrm{~B} 2$ & $\infty$ & L3 \\
\hline $\mathrm{T} 2$ & $\approx$ & $\mathrm{B} 2$ & & & & R3 & $\infty$ & T3 & $\mathrm{B} 2$ & $\infty$ & T3 \\
\hline L2 & $\approx$ & L3 & & & & R3 & $\infty$ & B3 & $\mathrm{B} 2$ & $\infty$ & L2 \\
\hline L2 & $\approx$ & $\mathrm{R} 2$ & & & & R3 & $\infty$ & $\mathrm{T} 2$ & $\mathrm{~B} 2$ & $\infty$ & R2 \\
\hline R2 & $\approx$ & L2 & & & & R3 & $\infty$ & L2 & $\mathrm{R} 2$ & $\infty$ & $\mathrm{T} 2$ \\
\hline R2 & $\approx$ & R3 & & & & L3 & $\infty$ & $\mathrm{T} 3$ & $\mathrm{R} 2$ & $\infty$ & B3 \\
\hline $\mathrm{B} 2$ & $\approx$ & B3 & & & & L3 & $\infty$ & B3 & $\mathrm{R} 2$ & $\infty$ & L3 \\
\hline $\mathrm{B} 2$ & $\approx$ & R3 & & & & L3 & $\infty$ & $\mathrm{B} 2$ & $\mathrm{R} 2$ & $\infty$ & $\mathrm{B} 2$ \\
\hline & & & & & & L3 & $\infty$ & $\mathrm{R} 2$ & & & \\
\hline
\end{tabular}

Given the form (see Figure 4), the information and the specified rules are educed and the authorized and unauthorized routes are determined. In fact, this information is derived from the data obtained from sensors and cameras embedded at the intersection. The system announced traffic commands according to these data Also, given the fact that the operation (Sense) is performed at the beginning of the kilometer of that input area, and then the equipment is moving along the freeway line. In the next steps, the equipment that traversed the freeway route, after the outside of the range entered the second range that the sensor set is on the freeway depending on the time it was set, is a time unit-and speed. Until repeated the previous stages, and eventually we will have the extra data that has been collected on the corresponding freeway.

\subsection{Preparing Data from Intersection Modeling}

The information received from the sensors consisted of the name of the vehicle, the speed of the gear, the speed, the number, the position, the capacity of the transmission, the capacity of the road, the demand for access and the route moving. The data are transmitted by the sensors to the database and in every 30 seconds the information is updated. These data are stored in the database. Our goal is to extract data, which can be used to reduce the number of accidents and in particular, to determine the critical points and in the event of an incident, we will help to manage the traffic.

\subsection{How to Calculate Reliability}

In this research, we will label a failure rate to calculate reliability for each of the orders. Of course, all of these rates are dynamically designed in a model that is closer to reality. Equation (1) is used to assess reliability:

Equation (1)

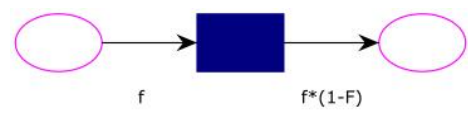


In the above equation, $\mathrm{f}$ is reliable and $\mathrm{F}$ is the error rate or refractive index, after each time in the event of a reliability error calculation, the new failure and reliability of the new event is calculated and updated the average.

\subsection{Create an Applicable Model}

In this paper, a colored petri nets and CPN Tools are used to create the applicable model.

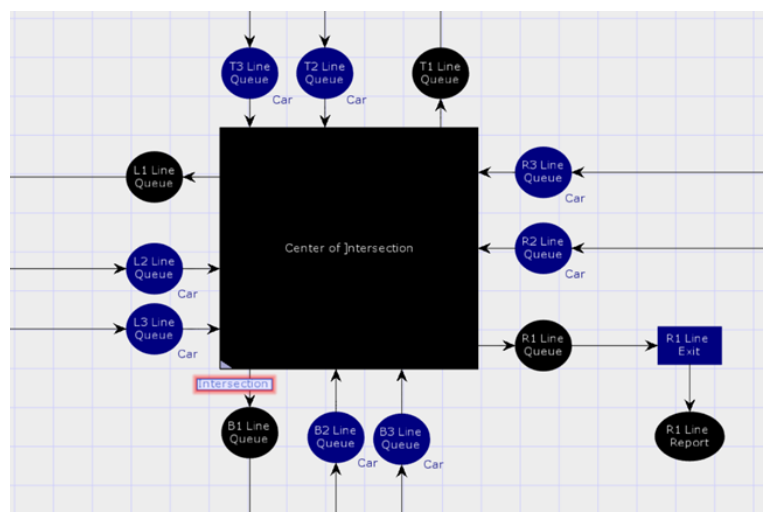

Figure 5 - Executable model of an urban intersection

Reliability assessment and response time done in the context of a specific architecture of the urban intersection conforming to (see Figure 4), using safety standards and urban traffic control with a failure rate label in each command issued by the intelligent monitoring system. (see Figure 5) shows the applicable model of a separate urban intersection.

\section{CASE STUDY}

In this paper, an example of the urban intersection (four-way) has been investigated for lack of complexity to demonstrate the feasibility and accuracy of the proposed method. Using the proposed method and simulation of the applicable model in the CPN Tools tool, we examine the metric of reliability and response time. The proposed approach is an intersection, which has features such as checking traffic, congestion, moving vehicles from source to destination, intelligent targeting-the traffic lights, and traffic management in the long run. In order to reach this important, the intersection is composed of sensors on the way, which, at the same time recorded the traffic in the Central server with due regard to the speed of the vehicle.
Table 2. shows the assumptions and standards defined for our study mode

\begin{tabular}{|l|l|l|l|}
\hline Time passing intersection & 3500 & 4000 & MS \\
\hline Reject time & 10 & 30 & MS \\
\hline Request time & 1500 & MS \\
\hline Time Out & 1500 & MS \\
\hline Chance of failure Every message & 0.08 & \\
\hline $\begin{array}{l}\text { Total number of intersection } \\
\text { vehicles }\end{array}$ & 10 & \\
\hline Thresholds for precursors (TH) & 4 & \\
\hline
\end{tabular}

In this stage, the goal of the modeling is to determine the cross-sectional and interpolated data of the equipment.

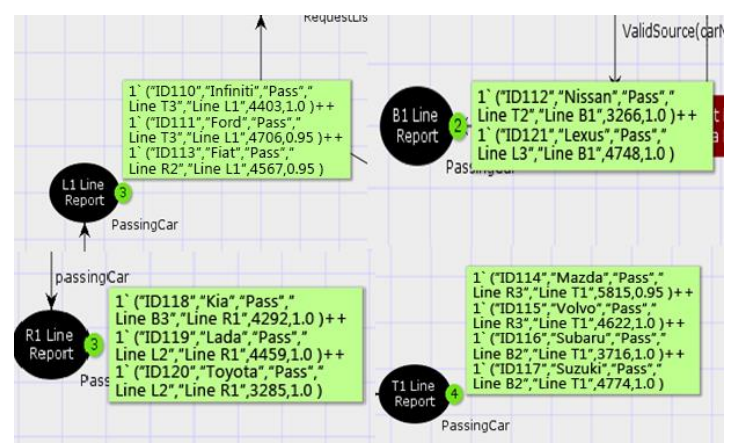

Figure 6. Executable model results from an urban intersection

In the following, to show the outputs (see Figure 6) of the performed model for one-time execution and the assumption of the entry of three cars per line, this is obtained in four directions of the intersection. To better understand the results of the model, we show the outputs for the execution specified in Table (3).

According to the results in Table (3), the mean reliability and response time for each separate line are calculated. Table (4) shows this possibility.

Table 3. Executable model results from an urban intersection

\begin{tabular}{|c|c|c|c|c|c|c|c|}
\hline $\begin{array}{c}\text { Inters } \\
\text { ection }\end{array}$ & $\begin{array}{c}\text { Vehicle } \\
\text { license plate }\end{array}$ & $\begin{array}{c}\text { Vehicle } \\
\text { name }\end{array}$ & Vehicle mode & $\begin{array}{c}\text { Source } \\
\text { ID }\end{array}$ & $\begin{array}{c}\text { Destinatio } \\
\text { n ID }\end{array}$ & $\begin{array}{c}\text { Respons } \\
\text { e time }\end{array}$ & Reliability \\
\hline \multirow{3}{*}{ L1 } & ID110 & Infinity & PASS & Line T3 & Line L1 & 4403 & 1.0 \\
\cline { 2 - 8 } & ID111 & Ford & PASS & Line T3 & Line L1 & 4706 & 0.95 \\
\cline { 2 - 9 } & ID113 & Fiat & PASS & Line R2 & Line L1 & 4567 & 0.95 \\
\hline \multirow{2}{*}{ B1 } & ID112 & Nissan & PASS & Line T2 & Line B1 & 3266 & 1.0 \\
\cline { 2 - 9 } & ID121 & Lexus & PASS & Line L3 & Line B1 & 4748 & 1.0 \\
\hline \multirow{3}{*}{ R1 } & ID118 & Kia & PASS & Line B3 & Line R1 & 4292 & 1.0 \\
\cline { 2 - 9 } & ID119 & Lada & PASS & Line L2 & Line R1 & 4459 & 1.0 \\
\hline \multirow{3}{*}{ T1 } & ID120 & Toyota & PASS & Line L2 & Line R1 & 3285 & 1.0 \\
\cline { 2 - 9 } & ID114 & Mazda & PASS & Line R3 & Line T1 & 5815 & 0.95 \\
\cline { 2 - 9 } & ID116 & Volvo & PASS & Line R3 & Line T1 & 4622 & 1.0 \\
\hline
\end{tabular}


International Journal of Computer Applications Technology and Research

Volume 7-Issue 01, 28-34, 2018, ISSN:-2319-8656

\begin{tabular}{|l|l|l|l|l|l|l|l|} 
& ID117 & Suzuki & PASS & Line B2 & Line T1 & 4774 & 1.0 \\
\hline
\end{tabular}

Table 4. Average results applicable model of a city intersection

\begin{tabular}{|c|c|c|}
\hline Intersection & $\begin{array}{c}\text { Response } \\
\text { time }\end{array}$ & Reliability \\
\hline L1 & 4558.66667 & 0.966666667 \\
\hline B1 & 4007 & 1 \\
\hline R1 & 4012 & 1 \\
\hline T1 & 4731.75 & 0.9875 \\
\hline
\end{tabular}

According to the results and insert in Table (4), we can draw graphs of the parameters studied.

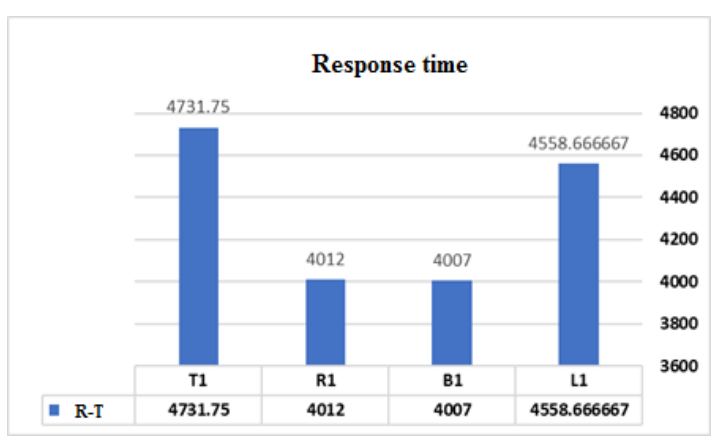

Figure 7. Response time of the applicable model from an urban intersection

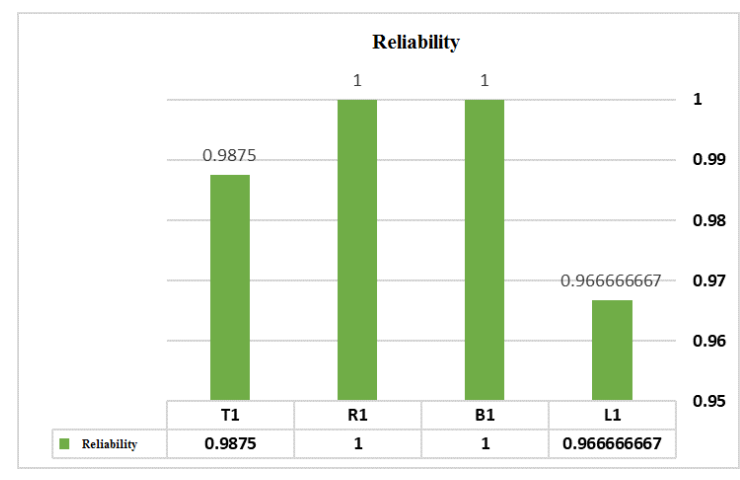

Figure 8. Reliability of the applicable model from an urban intersection

According to the results of the implementation of the model, reliability and response time are also obtained according to Table (5) of the proposed method in the total intersection.

Table 5. The average efficiency of the proposed method model

\begin{tabular}{|c|c|c|}
\hline \multirow{2}{*}{$\begin{array}{c}\text { Average } \\
\text { Total }\end{array}$} & Response time & Reliability \\
\cline { 2 - 3 } & 4327.354167 & 0.988541667 \\
\hline
\end{tabular}

\section{REFERENCES}

[1] A.Pande, and M.Abdel-Aty. "Assessment of freeway traffic parameters leading to lane-change related collisions." Accident Analysis \& Prevention 38.5 (2006): 936-948.

Table 6. Incoming Line Intersection

\begin{tabular}{|c|c|c|c|}
\hline Title & $\begin{array}{c}\text { Queue } \\
\text { length }\end{array}$ & Title & $\begin{array}{c}\text { Queue } \\
\text { length }\end{array}$ \\
\hline Line B2 & 2 & Line L3 & 1 \\
\hline Line B3 & 1 & Line R2 & 1 \\
\hline Line L2 & 2 & Line R3 & 2 \\
\hline Line T2 & 1 & Line T3 & 2 \\
\hline
\end{tabular}

The graph of Table (6) is shown in (see Figure 8)

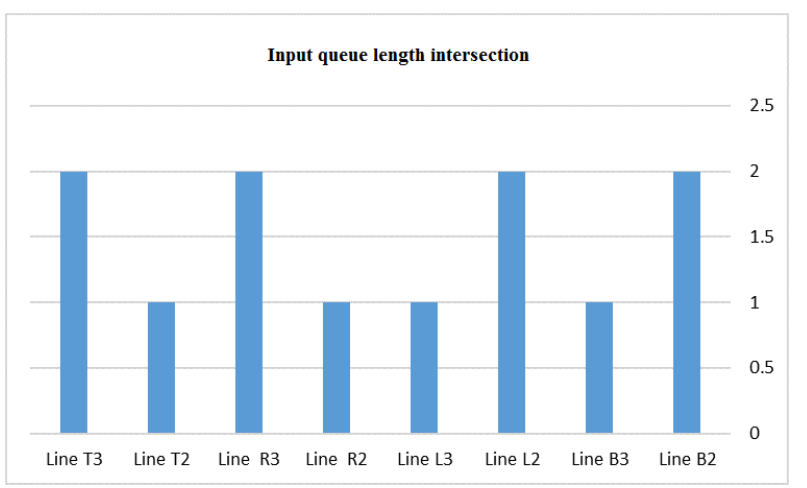

Figure 9. Incoming queue length intersection of applicable model from an urban intersection

\section{CONCLUSION} real-time using colored petri nets in order to evaluate the model conditions. And the possibility was also found in the designed model that can dynamically evaluate the flexibility of inputs and conditions. The design of the model was compared to the evaluation of reliability parameters and response time. The results showed that these systems have the ability to execute and can be implemented in reality if the veloped countries are progressive in this regard and they can manage secure traffic and congestion control at the

One of the advantages of the proposed method is that we can evaluate the system before the implementation phase and spending time and money. One of the suggestions for future activities is to evaluate the traffic monitoring and control systems according to the behavior of the agents. 
[2] Gharooni fard, G., Moein darbari, F., Deldari, H., Morvaridi, A., Scheduling of scientific workflows using a chaos- genetic algorithm, Procedia Computer Science, Elsevier, Vol. 1, No.1, pp. 1445- 1454, (2010).

[3] H.Ahmad Tavassoli, et al. "Modelling the impact of traffic incidents on travel time reliability." Transportation Research Part C: Emerging Technologies 65 (2016): 4960.

[4] H.Becker, R. Koziolek, "Model Based Performance Prediction with the Palladio Component Model", WOSP'07, ACM, Buenos Aires, Argentina, no. 4, (2006), pp. 54-65.

[5] H. Motameni, A.Movaghar, M. Siasifar, "Analytical evaluation on Petri net by using Markov chain theory to achieve optimized Model". World Appl. (2008),Sci. J. 3 (3) 504-513.

[6] K. Jensen, "Colored Petri Nets: Basic Concepts, Analysis Methods and Practical Use", EATCS Monographs on Theoretical Computer Science, Vol. 29, No .2,(2007), pp70-120.

[7] L.M. Kristensen , L.Wells, K. Lensen., "Coloured Petri Nets and CPN Tools for modeling and validation of concurrent systems," International Journal on Software Tools for Technolog Transfer (STTT), no. Springer Berlin / Heidelberg,(2007), pp. 213-254.

[8] M.Ahmed, et al. "Exploring a Bayesian hierarchical approach for developing safety performance functions for a mountainous freeway." Accident Analysis \& Prevention 43.4 (2011): 1581-1589.

[9] P.T. Martin, Y. Feng, and X. Wang, Detector technology evaluation. No. MPC Report No. 03-154. MountainPlains Consortium, (2003).

[10] 10) R.Yu, and M. Abdel-Aty. "Multi-level Bayesian analyses for single-and multi-vehicle freeway crashes." Accident Analysis \& Prevention 58 (2013): 97-105.

[11] R. Yu, and M. Abdel-Aty, "Utilizing support vector machine in real-time crash risk evaluation." Accident Analysis \& Prevention 51 (2013): 252-259.

[12] R.Yu, M.Abdel-Aty, M.Ahmed." Bayesian random effect models incorporating realtime weather and traffic data to investigate mountainous freeway hazardous factors." Accident Analysis \& Prevention 50 (0), 371-376.(2013)

[13] X.Sun, et al. "Research on Traffic State Evaluation Method for Urban Road." Intelligent Transportation, Big Data and Smart City (ICITBS), 2015 International Conference on. IEEE, (2015).

[14] X.Xiujuan, et al. "A novel algorithm for urban traffic congestion detection based on GPS data compression." Service Operations and Logistics, and Informatics (SOLI), (2016) IEEE International Conference on. IEEE. 